\title{
Independent Population Validation of the Belin/Ambrósio Enhanced Ectasia Display: Implications for Keratoconus Studies and Screening
}

\author{
${ }^{1}$ Ovette F Villavicencio, ${ }^{2}$ Fatimah Gilani, ${ }^{3}$ Maria A Henriquez, ${ }^{4}$ Luis Izquierdo Jr \\ ${ }^{5}$ Renato R Ambrósio Jr, ${ }^{6}$ Michael W Belin
}

\begin{abstract}
With advances in technology and imaging, finding diagnostic criteria that are both sensitive and specific for keratoconus while using the latest corneal imaging modalities is paramount. The Belin/Ambrósio enhanced ectasia display final ' $D$ ' index, tested on an independent population, illustrated excellent false positive rates for refractive screening while eliminating $99 \%$ of keratoconus corneas. A false positive rate of $0 \%$ is achieved with a final ' $D$ ' of 2.69 , meeting the more stringent criteria for treatment studies.
\end{abstract}

Keywords: Keratoconus, Ectasia, Tomography, Cornea, Imaging, Refractive surgery, Cross-linking, Scheimpflug, Placido, Topography.

How to cite this article: Villavicencio OF, Gilani F, Henriquez MA, Izquierdo L Jr, Ambrósio RR Jr, Belin MW. Independent Population Validation of the Belin/Ambrósio Enhanced Ectasia Display: Implications for Keratoconus Studies and Screening. Int J Kerat Ect Cor Dis 2014;3(1):1-8.

\section{Source of support: Nil}

Conflict of interest: Drs Ambrosio and Belin are consultant to OCULUS GmbH.

\section{INTRODUCTION}

Prior to refractive surgery the diagnosis of keratoconus was based on the distortion of the anterior cornea and the resultant decrease in visual acuity. Treatments, both surgical and nonsurgical were based on visual loss, a fairly late sequelae. Steep and distorted keratometry, corneal striae and scarring,

\footnotetext{
${ }^{1}$ Resident (PGY-4), ${ }^{2}$ Resident (PGY-3), ${ }^{3}$ Associate Clinical Professor, ${ }^{4,6}$ Professor, ${ }^{5}$ Associate Professor

1,2,6 Department of Ophthalmology and Vision Science University of Arizona, Tucson, Arizona, USA

${ }^{3,4}$ Department of Research, Oftalmosalud Instituto de Ojos Lima, Peru

${ }^{5}$ Department of Ophthalmology, Federal University of São Paulo, São Paulo; Rio de Janeiro Corneal Tomography and Biomechanics Study Group, Rio de Janeiro, Brazil
}

Corresponding Author: Michael W Belin, Professor Department of Ophthalmology and Vision Science, University of Arizona, 4232 West Summer Ranch Place, Marana, Arizona USA, Phone: +15185271933, e-mail: mwbelin@aol.com and loss of best corrected spectacle visual acuity were hallmarks of keratoconus and represent advanced manifestations of the ectatic process. ${ }^{1,2}$ Refractive surgery, however, put new stresses on the cornea and revealed what was previously viewed as 'normal' could, if biomechanically stressed, could undergo progressive ectatic change. ${ }^{3-6}$

More recently, treatments, such as cross-linking, have moved the timing of intervention from the late stage to earlier in the disease process. Cross-linking is being utilized to stabilize an ectatic cornea at the earliest signs of visual loss. ${ }^{7-9}$ Conceivably, however, the most efficacious timing may be prior to any visual loss, which requires an accurate diagnosis at a much earlier stage than was previously necessary or possible. ${ }^{10}$

Quoted figures and estimates of keratoconus fall between 50 and 230 per 100,000 with variations across different populations and complicated by different criteria used for the diagnosis. ${ }^{2}$ The challenge most face is diagnosing early ectatic disease before apparent clinical manifestations. Although almost all ophthalmologists routinely screen patients prior to refractive surgery using corneal imaging, many have difficulty identifying abnormalities indicative of early keratoconus. ${ }^{11}$ The problem is amplified by the lack of a concise definition of early, suspected or 'form fruste' keratoconus.

Marc Amsler in 1938 used photographic placido disk to describe early corneal topographic changes and coined the term 'form fruste keratoconus'. ${ }^{12,13}$ 'Subclinical keratoconus' is described as early preclinical keratoconus only detectable by diagnostic examinations such as corneal tomography. Topographic and/or tomographic features suspicious for keratoconus are described as 'keratoconus suspect' especially if there are no clinical signs or history of keratoconus.

The Amsler-Krumeich classification of keratoconus is based on disease evolution. The severity of keratoconus is classified into 4 stages with the central $\mathrm{K}$ value having significant importance. Mean central $\mathrm{K}$ readings of $<48$ Diopters (D) in addition to eccentric steepening and mild myopia and astigmatism indicate stage I disease. Stage II disease has mean central $\mathrm{K}$ readings $\angle 53 \mathrm{D}$ and increasing myopia and astigmatism with absence of scarring. Stage III disease has mean central $\mathrm{K}$ readings of $>53 \mathrm{D}$, myopia and 
astigmatism from 8 to $10 \mathrm{D}$ and minimum corneal thickness of 300 to 400 microns. Finally, stage IV disease is indicated by central corneal scarring with mean central $\mathrm{K}$ readings $>$ $55 \mathrm{D}$ with unmeasurable refractions. ${ }^{14}$

There are several other index-based systems that utilize Placido-based topographic criteria. These indices include: Pathfinder Corneal Analysis, keratoconus percentage index (KISA\%) ${ }^{15}$ and KPI and KCI indices from Maeda and Klyce, ${ }^{16,17}$ and I-S value. ${ }^{18}$

Rabinowitz utilized computer-assisted videophotokeratoscopy to devise indices used in an attempt to identify early keratoconus. ${ }^{15}$ These indices were central keratometry (K), inferior-superior (I-S), and the keratoconus percentage index from each eye examined under videokeratograph. ${ }^{18}$ The KISA index was derived from 2 additional indices besides central $\mathrm{K}$ and I-S, the astigmatism index (AST) which quantifies the degree of regular corneal astigmatism, and SRAX index, an expression of the irregular astigmatism. ${ }^{15}$

Maeda and Klyce ${ }^{16}$ utilized the keratoconus prediction index (KPI) a calculation from 8 quantitative videokeratography-derived indices. These indices include: SimK1 (major axis), SimK2 (minor axis), surface asymmetry index, differential sector index, opposite sector index, center/ surround index, and analyzed area. ${ }^{16}$

All of the above, however, were limited to imaging the anterior corneal surface and additionally relied on a single central corneal thickness value. Further advances in technology and imaging devices, including but not limited to rotating Scheimpflug optical cross-sectional analysis, confocal microscopy, optical coherence tomography, very high-frequency ultrasound, and biomechanical analysis have added additional diagnostic criteria to assist the clinician in diagnosing ectatic disease. ${ }^{19,20}$

The Belin/Ambrósio Enhanced Ectasia Display (BAD) was initially released for the Pentacam (OCULUS GmbH, Wetzlar Germany) in 2008. The display was designed to combine elevation data from the anterior and posterior cornea with pachymetric data in an effort to provide a more comprehensive display for keratoconus and ectasia screening. The initial release (BAD 1) showed anterior and posterior elevation with a standard $8 \mathrm{~mm}$ best fit sphere (BFS) reference surface, with an enhanced reference surface (ERS), and the numerical change between the two. The enhanced reference surfaces utilizes the standard BFS but modifies it by eliminating a variable 3.5 to $4 \mathrm{~mm}$ zone surrounding the thinnest point on the cornea. The exact exclusion zone size is determined by the astigmatism magnitude and is not operator selected. In a normal or keratoconic population (i.e. nonsurgically altered) the ERS is flatter than the standard BFS and results in an amplification of positive elevations. Since, the exclusion zone surrounds the thinnest portion of the cornea, eyes with significant ectasia (positive elevation) have a greater flattening of the ERS compared to the standard BFS, while normal eyes have an ERS that more closely mimics the standard BFS. The numerical difference between the elevation data going from the BFS to the ERS should therefore show greater change in eyes with ectasia. The BAD 1 also displayed the full pachymetric map as well as two pachymetric progression graphs, the 'corneal thickness spatial profile' (CTSP) and the 'percentage thickness increase' (PTI). The BAD 1 also displayed the following individual parameters; corneal thickness at the apex and thinnest point, distance between the apex and thinnest point, progression index (minimal, average and maximum) and a display quality check.

The second release (BAD II) (2010) reported the standard deviations from a large normative database of five parameters Df (change in anterior elevation from BFS to ERS), $\mathrm{Db}$ (change in posterior elevation), Dp (pachymetric progression), Dt (pachymetric value at the thinnest point), and Dy (vertical displacement of the thinnest point from the apex). These five parameters were then utilized in a regression analysis with the normative database and a known keratoconic database. The final ' $\mathrm{D}$ ' represents how the examined cornea varies from 'normal' when compared to the keratoconic group.

The current version (BAD III) (2012) (Fig. 1) added four additional parameters to the original regression analysis $\left(\mathrm{K}_{\max }\right.$, anterior and posterior elevation at the thinnest point and Ambrósio relational thickness maximum ( $\mathrm{ART}_{\max }$ ). $\mathrm{ART}_{\text {max }}$ is the maximal progression index divided by the thinnest pach. Additional individual parameters, not utilized in the regression analysis, were also added; steep and flat simulated keratometry ( $\mathrm{K} 1$ and $\mathrm{K} 2$ ) and $\mathrm{Q}$ value. The final ' $\mathrm{D}$ ' was further refined with additional testing against a population of so-called unilateral or highly asymmetric keratoconic patients.

The current study seeks to validate the BAD III using two new populations (both normative and keratoconic) that were not used in the original regression analysis (i.e. independent retest population) and to evaluate both individual $\mathrm{BAD}$ parameters as well as the final ' $D$ ' in their performance for both screening (i.e. exclusion) potential (e.g. refractive surgery screening) and for studies (i.e. inclusion) on keratoconus (e.g. cross-linking).

\section{MATERIALS AND METHODS}

The validation normative database consisted of 341 adults (682 eyes) details of which have been previously described. ${ }^{21}$ All patients had a normal ocular examination, a best corrected visual acuity of 20/20 or better and no family history of ectatic disease. Only simple and compound myopic patients were 


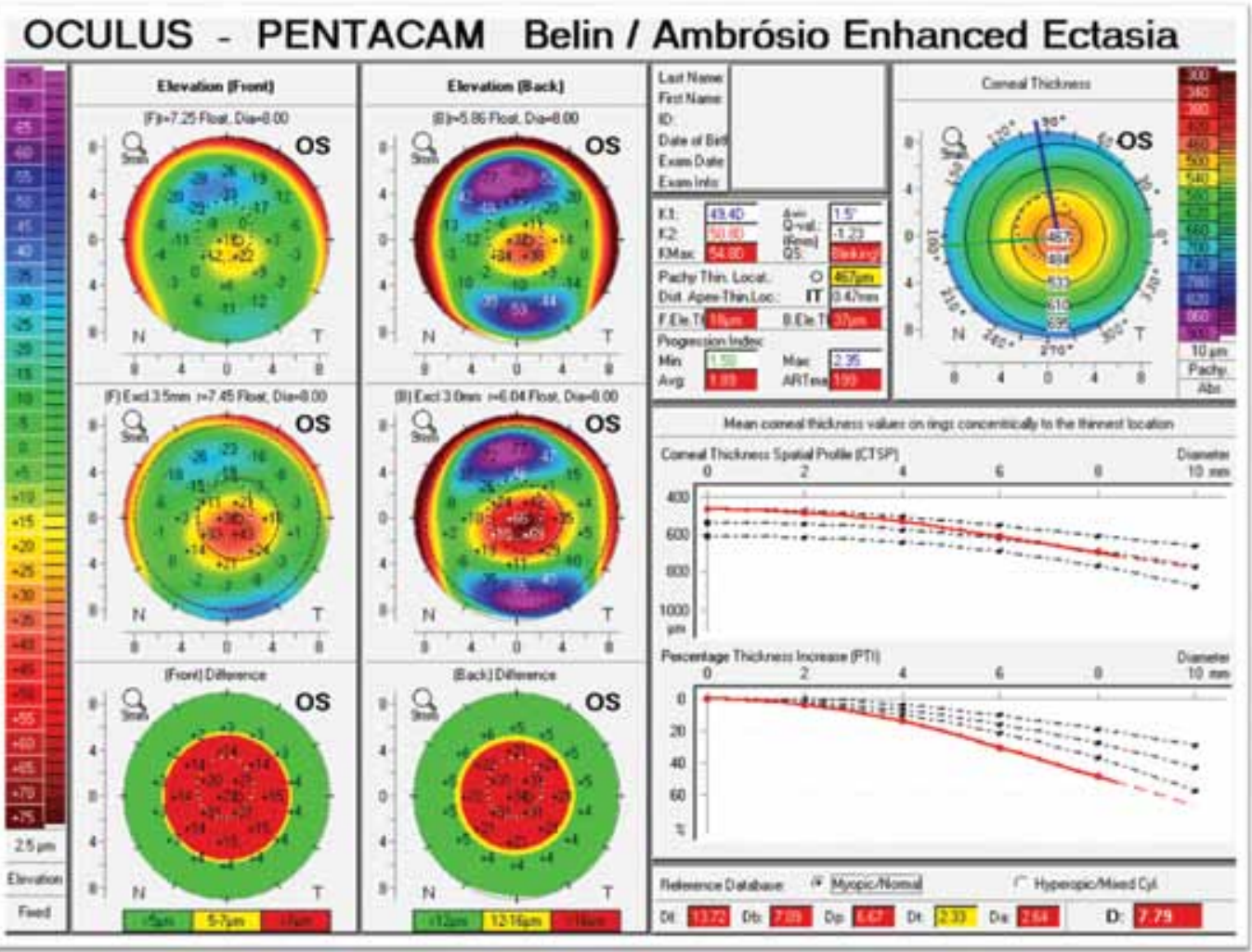

Fig. 1: BAD III sample display of keratoconus. Both anterior and posterior elevation maps show positive islands of elevation (upper elevation maps) that are amplified with the enhanced reference surface (middle elevation maps) and the lower display shows that the difference is significant. The upper-right pachymetric map reveals a thin cornea (thinnest point 467) with highly abnormal pachymetric progression graphs (both CTSP and PTI). Other individual parameters that are flagged as abnormal include $\mathrm{K}_{\max }$, Front and Back elevation at the thinnest point, $\mathrm{Pl}_{\mathrm{avg}}$, and $\mathrm{ART}_{\text {max }}$. The final ' $\mathrm{D}$ ' for this cornea is 7.79 .

included (range $-0.5 \mathrm{D}$ to $-10 \mathrm{D}$ ). Patients were included whether they proceeded to refractive surgery or not. All exams were independently examined by two fellowship trained refractive surgeons (MWB and RA). All patients had at least 3 years of clinically uneventful follow-up. Clinically uneventful follow-up was defined as no loss of one or more lines of vision, or an increase in astigmatism by $0.5 \mathrm{D}$ or the need for an enhancement. All exams had at least $9 \mathrm{~mm}$ of coverage with a minimum of $8 \mathrm{~mm}$ nonextrapolated data and an internal Pentacam quality check indicating no issues. One eye per patient was randomly selected for the initial analysis.

To minimize bias, the keratoconic data base ( 74 patients (147 eyes)) was provided by an outside research department, Oftalmosalud Instituto de Ojos (Lima, Peru). All examining physicians at the institute are fellowship trained corneal specialists (MAH, LI). They were contacted based on a prior published paper describing a large keratoconic patient population..$^{22}$ Instructions were limited to providing a keratoconic database with at least $8 \mathrm{~mm}$ of coverage on Pentacam examination. Complete U12 files were provided. This database was then reviewed by MWB and RA only to exclude exams that did not meet quality standards. Eyes that had any prior surgical alteration were eliminated.

Individual patient data were imported into an Excel file using a specially designed extraction program. The following parameters were analyzed: Minimal central corneal thickness, $\mathrm{K}_{\max }$, Df (change in front elevation from standard BFS to enhanced BDS), Db (change in back elevation from standard BFS to enhanced BFS), Front elevation at the thinnest point, Back elevation at the thinnest point, PIavg (pachymetric progression index), $\mathrm{ART}_{\text {max }}$ (Ambrosio relational thickness), final ' $D$ '. All elevation values were referenced against a BFS taken from the central $8 \mathrm{~mm}$ of the cornea. The final ' $\mathrm{D}$ ' is a derived parameter based on a regression analysis designed to distinguish between normal and ectatic corneas and was based on a prior (i.e. initial) study population..$^{23,24}$ Each individual parameter and the final ' $D$ ' were then analyzed for screening potential (e.g. refractive surgical screening) using a one-sided $95 \%$ and $97.5 \%$ confidence interval (i.e. a $5 \%$ and $2.5 \%$ false positive rate) and for inclusion potential for keratoconic studies using a 95 and $97.5 \%$ capture rates and a $99.9 \%$ normal exclusion rate $(0.1 \%$ false positive). Finally the screening values and inclusion values were compared.

\section{RESULTS}

Six hundred eighty-two eyes of 341 patients ( 177 female/164 male) were analyzed for the normative database with an age range of 18 to 68 years. Racial data were not identifiable, but the vast majority of the study population was white. One 
eye of each patient was randomly selected as each eye is not viewed as an independent variable (details of this normative database were previously published (J Cat Refract Surg 2013;39(11):1707-1712).

One hundred forty-seven eyes of 74 keratoconic patients were analyzed with an age range of 12 to 58 years. All patients were screened at the Oftalmosalud Instituto de Ojos,

Table 1: Mean, standard deviation, and range for 9 tomographic parameters in normal and keratoconus population (all elevation values referenced of a BFS from the central $8.0 \mathrm{~mm}$ zone) (Pentacam, OCULUS GmbH)

\begin{tabular}{|c|c|c|c|}
\hline & & Normal & Keratoconus \\
\hline Eyes & & 341 & 147 \\
\hline \multirow[t]{3}{*}{ Minimal pach } & Mean & 539 & 473 \\
\hline & Std. & 30.7 & 50.3 \\
\hline & Range & 459 to 622 & 156 to 564 \\
\hline \multirow[t]{3}{*}{$\mathrm{K}_{\max }$} & Mean & 45.3 & 54.9 \\
\hline & Std. & 1.47 & 8.23 \\
\hline & Range & 40.9 to 51.3 & 44.8 to 106.7 \\
\hline \multirow[t]{3}{*}{ Df } & Mean & -0.07 & 9.2 \\
\hline & Std. & 1.01 & 6.92 \\
\hline & Range & -1.86 to 4.02 & -1.1 to 37.7 \\
\hline \multirow[t]{3}{*}{$\mathrm{Db}$} & Mean & -0.10 & 6.69 \\
\hline & Std. & 0.9 & 5.60 \\
\hline & Range & 1.5 to 3.77 & -0.52 to 40.5 \\
\hline \multirow[t]{3}{*}{ Elev front thin } & Mean & 1.7 & 18.3 \\
\hline & Std. & 1.6 & 11.0 \\
\hline & Range & -3.0 to 8.0 & -3 to 52 \\
\hline \multirow[t]{3}{*}{ Elev back thin } & Mean & 4.1 & 39.8 \\
\hline & Std. & 4.10 & 25.59 \\
\hline & Range & -8 to 18 & -3.0 to 162 \\
\hline \multirow[t]{3}{*}{$\mathrm{Pl}_{\text {avg }}$} & Mean & 0.92 & 2.09 \\
\hline & Std. & 0.13 & 1.97 \\
\hline & Range & 0.53 to 1.36 & 0.82 to 22.8 \\
\hline \multirow[t]{3}{*}{$\mathrm{ART}_{\max }$} & Mean & 473 & 209.9 \\
\hline & Std. & 84.3 & 81.5 \\
\hline & Range & 253 to 893 & 4 to 557 \\
\hline \multirow[t]{3}{*}{ D } & Mean & 0.69 & 7.49 \\
\hline & Std. & 0.58 & 5.56 \\
\hline & Range & -1.25 to 2.61 & 1.55 to 54.77 \\
\hline
\end{tabular}

Table 2: Sample refractive screening using 5 and $2.5 \%$ false positive rates with accompanying percentage of keratoconus eyes included

\begin{tabular}{lll}
\hline & $\begin{array}{l}95 \% \mathrm{Cl} / \mathrm{KCN} \\
\text { included(\%) }\end{array}$ & $\begin{array}{l}97.5 \% \mathrm{Cl} / \mathrm{KCN} \\
\text { included(\%) }\end{array}$ \\
\hline Minimal pach & $489 / 38.1 \%$ & $480 / 43.5 \%$ \\
$\mathrm{~K}_{\max }$ & $47.7 / 9.5 \%$ & $48.1 / 10 \%$ \\
$\mathrm{Df}$ & $1.66 / 6.8 \%$ & $2.14 / 10 \%$ \\
$\mathrm{Db}$ & $1.63 / 11 \%$ & $2.05 / 15 \%$ \\
Elev front thinnest & $4.0 / 4.8 \%$ & $5.0 / 6.1 \%$ \\
Elev back thinnest & $12.0 / 6.1 \%$ & $13.0 / 6.1 \%$ \\
$\mathrm{Pl}_{\text {avg }}$ & $1.14 / 4.1 \%$ & $1.18 / 6.1 \%$ \\
$\mathrm{ART}_{\max }$ & $353 / 4.8 \%$ & $332 / 8.8 \%$ \\
Final D & $1.65 / 0.7 \%$ & $1.88 / 0.7 \%$ \\
\hline
\end{tabular}

Lima, Peru and are assumed to be native. Both eyes (one eye received cross-linking and was excluded) were included in the analysis, since, the purpose of the study was to determine the potential to identify ectatic disease.

The mean, standard deviation and range of each parameter are shown in Table 1. The actual distribution for each parameter and final ' $\mathrm{D}$ ' are shown in shatter graphs (Figs 2A to I). The insert shows only the region of overlap between the normal and keratoconic population.

Refractive surgery screening entails examining an assumed normal population to identify potential at risk patients. This often involves not only trying to identify patients with true disease, but also identifying outliers, whom may or may not have disease, but are assumed to be at higher risk or in whom risk data analysis is lacking. We arbitrarily selected at 95 and $97.5 \%$ one-sided confidence interval (accepting a $5 \%$ and $2.5 \%$ false positive rate) for the normal population as representative of a reasonably risk adverse screening. Each of the 8 individual parameters and the final ' $D$ ' are shown in Table 2 with both the representative screening gates and the percentage of keratoconus patients that would be incorrectly included as 'normal' (false negatives). The worst performing individual parameter was minimal corneal thickness which would include up to $43.5 \%$ of the keratoconic population, while the best performing parameter was the final ' $\mathrm{D}$ ' which included $<1 \%$ of the keratoconic group.

Conversely, one can examine a 95 and $97.5 \%$ keratoconus capture rate. The results are shown in Table 3 with the associated gates and percentage of normal eyes that would be included (false positives). The worst performing individual parameter in this scenario is the front elevation at thinnest with a false positive rate of $98.1 \%$, and the best performing parameter is the final ' $\mathrm{D}$ ' with a zero false positive rate at the $95 \%$ keratoconic capture rate.

Treatment studies, as opposed to screening to exclude disease, requires a totally different set of parameters to insure that the majority of those entered into a trail actually have the disease being studied. Table 4 shows the keratoconus capture

Table 3: Percentage of normals included for each parameter, if 95 and $97.5 \%$ of keratoconic eyes are included

\begin{tabular}{lll}
\hline & $\begin{array}{l}95 \% \mathrm{Cl} / \mathrm{normal} \\
\text { included(\%) }\end{array}$ & $\begin{array}{l}97.5 \% \mathrm{Cl} / \mathrm{normal} \\
\text { included(\%) }\end{array}$ \\
\hline Minimal pach & $538 / 49 \%$ & $558 / 73 \%$ \\
$\mathrm{~K}_{\max }$ & $46.1 / 31 \%$ & $45.3 / 55 \%$ \\
$\mathrm{Df}$ & $1.43 / 8.2 \%$ & $-0.2 / 50 \%$ \\
$\mathrm{Db}$ & $1.0 / 13 \%$ & $0.48 / 23 \%$ \\
Elev front thinnest & $4.3 / 3.5 \%$ & $-1.7 / 98.1 \%$ \\
Elev back thinnest & $11.3 / 5.7 \%$ & $6.3 / 25 \%$ \\
$\mathrm{PI}_{\text {avg }}$ & $1.17 / 3.2 \%$ & $1.08 / 12 \%$ \\
$\mathrm{ART}_{\max }$ & $354 / 5.3 \%$ & $376 / 11 \%$ \\
Final D & $2.69 / 0 \%$ & $2.26 / 0.6 \%$ \\
\hline
\end{tabular}


Table 4: Percentage of keratoconus eyes included for each parameter, if $99.9 \%$ of normal eyes are excluded

\begin{tabular}{llllllllll}
\hline & $\begin{array}{l}\text { Minimal } \\
\text { pach }\end{array}$ & $K_{\max }$ & $D f$ & $D b$ & $\begin{array}{l}\text { Elev front } \\
\text { thinnest }\end{array}$ & $\begin{array}{l}\text { Elev back } \\
\text { thinnest }\end{array}$ & $P_{\text {avg }}$ & ART $\max$ & Final D \\
\hline $99.9 \%$ normal exclusion & 459 & 51.3 & 4.02 & 3.77 & 8 & 18 & 1.36 & 253 & 2.61 \\
$\% \mathrm{KCN}$ capture & $33 \%$ & $63 \%$ & $74 \%$ & $65 \%$ & $87 \%$ & $87 \%$ & $85 \%$ & $71 \%$ & $95 \%$ \\
\hline
\end{tabular}
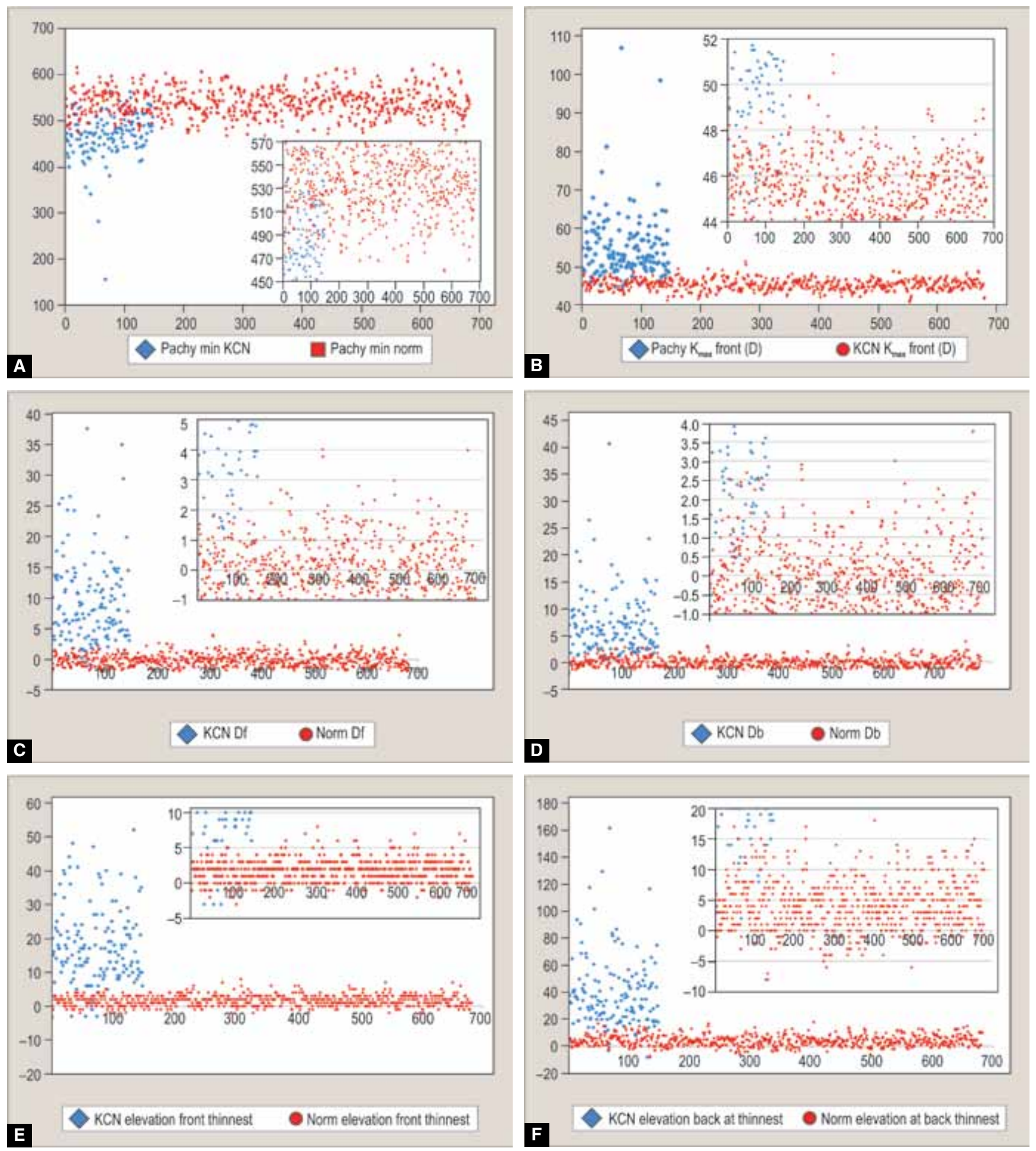

Figs 2A to $F$

Contd... 


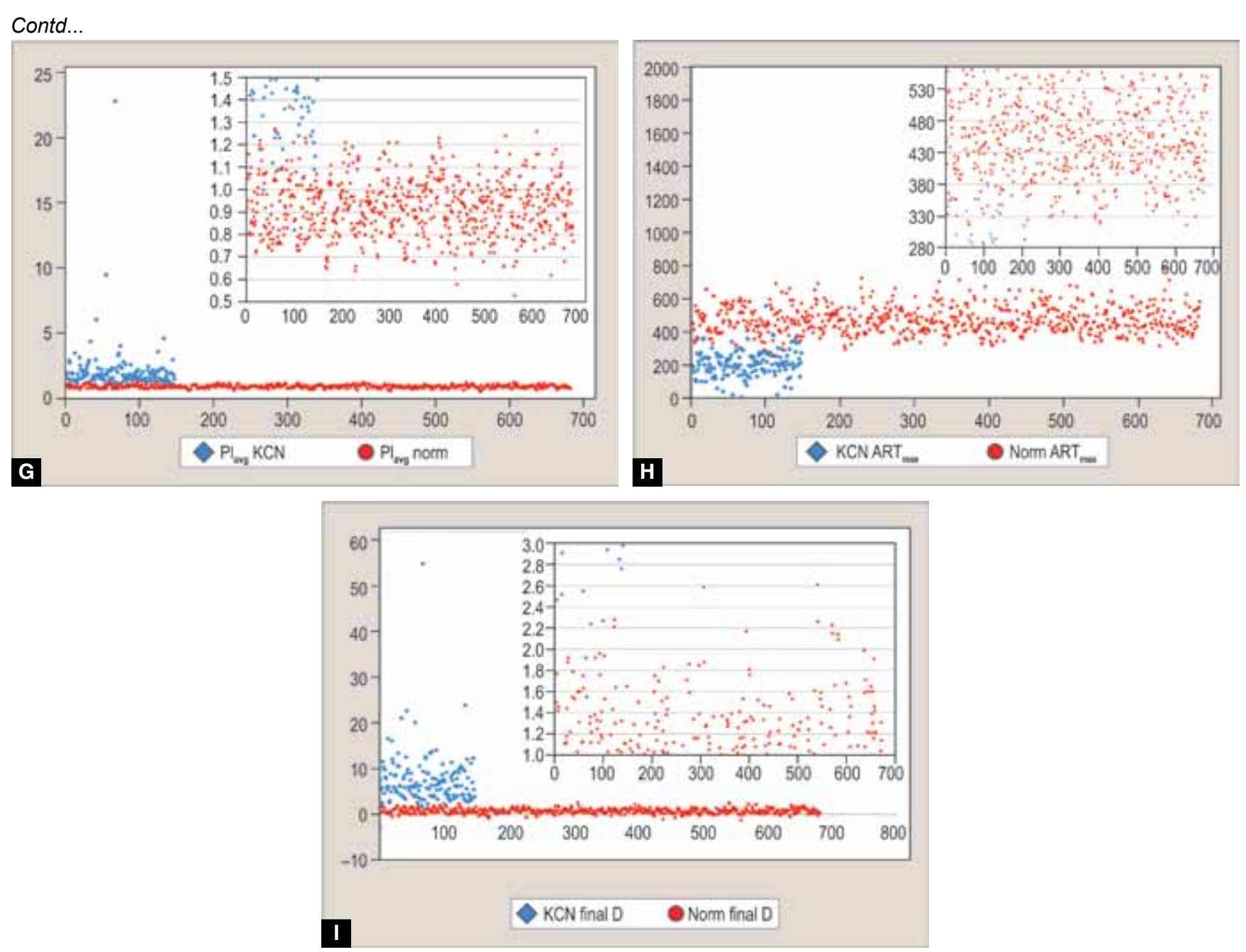

Figs 2A to I: Individual Scatter graphs of the 8 individual parameters minimal pachymetry, $\mathrm{K}_{\max }$, Df (front elevation difference), Db (back elevation difference), elevation front thinnest, elevation back thinnest, $\mathrm{Pl}_{\mathrm{avg}}, \mathrm{ART}_{\max }$ ) and the final overall reading ' $\mathrm{D}$ '. The smaller insert graphs display only the area of overlap between the normative population (red) and the keratoconus population (blue)

percentage if one excludes $99.9 \%$ ( $0.1 \%$ false positive) of normal patients. Minimal pachymetry is the worst performing parameter, capturing only one-third of the keratoconic group, while the final ' $\mathrm{D}$ ' performs the best with a $95 \%$ capture rate.

\section{DISCUSSION}

An optimal screening test for refractive surgery should be relatively simple and quick to perform and to be able to identify 'abnormals' with an acceptable degree of false positives. Rates of keratoconus range from $1 / 400$ to $1 / 2,000$, the latter being oft quoted for an US population. These rates probably underestimate the frequency of keratoconus seen in a refractive screening population which tends to pre-select myopes, contact lens wearers and individuals dissatisfied with their vision, all of which are more common in keratoconic individuals. ${ }^{25} \mathrm{We}$ arbitrarily selected a 95 and $97.5 \%$ one-sided confidence interval for the normal population and reported the percentage of a keratoconic population that would be included (false negative) (Table 2) and a 95 and $97.5 \%$ keratoconus detection rate and report the accompanying percentage of false positives (i.e. normals) associated with that detection rate (Table 3 ). These values will also vary based on geographic variation in the incidence of keratoconus as well as individual surgeon's choice for screening parameters. The final ' $D$,' based on a regression analysis of all the parameters, is the best performing screening parameter with values of $1.65 / 1.88$ associated with a 95 and $97.5 \%$ confidence interval with an acceptable false negative rate of less than $1 \%$.

As opposed to screening normal patients for disease, inclusion studies for treating keratoconus (e.g. cross-linking) require a completely different set of parameters. Unfortunately, this has typically not been done in studies treating early or 'form-fruste' disease. ${ }^{26}$ For example, if one chooses the $95 \%$ keratoconus detection rate the accompanying false positive rate of the best performing individual parameter $\left(\mathrm{ART}_{\max }\right)$ is $5 \%$. If you used this parameter to determine entrance into a clinical trial, then you would have 49 normal patients misdiagnosed as abnormal for every true keratoconic 
(assuming a 1/1,000 incidence), making treatment efficacy claims problematic. Only the final ' $\mathrm{D}$ ' appears to have adequate discriminatory potential to screen for treatment (i.e. inclusion) studies. In practice, most parameters would likely perform better taking into account additional screening by family history and perhaps other ancillary testing.

This study analyzed the ability of the BAD display to separate a normal population from a keratoconic population. The study utilized two new databases that were not utilized in the initial regression analysis (retest population). The study validates the BAD's final ' $\mathrm{D}$ ' as the most useful of the nine parameters tested and the only one with sufficient discriminatory potential to serve both for refractive screening and for treatment protocols (though at very different gates). Separating a normal population from a known keratoconic population is very different from assessing risk or screening for potential post-LASIK ectasia. Surgical patients at increased risk may not have currently measurable risk factors or may only exhibit evidence of subclinical keratoconus (i.e. normal anterior surface in the presence of posterior ectasia and/or abnormal pachymetric progression). Our keratoconic study population was heavily weighted toward moderate disease with observable anterior changes with an average topographical keratoconus classification (TKC) score of 1.94 and only 6/147 eyes classified as early or subclinical disease. The TKC score relies solely on anterior corneal shape and is designed to mimic similar placido based keratoconus classifications. ${ }^{27,28}$ Future work with earlier and/or subclinical disease should allow for further refinement.

\section{CONCLUSION}

The Belin/Ambrósio enhanced ectasia display final ' $D$ ' shows excellent potential for use in both refractive screening and for treatment inclusion studies albeit at significantly different gates.

The final ' $\mathrm{D}$ ' is a product of a multivariate regression analysis and validation tested was performed using two (normal and keratoconic) independent retest populations. No tested individual parameters performed sufficiently to function in a similar manner. Final ' $D$ ' values of 1.65 and 1.88 were associated with a 5 and $2.5 \%$ false positive rate, reasonable for refractive screening while eliminating over 99\% of known keratoconus corneas, while a final ' $\mathrm{D}$ ' of 2.69 had a $0 \%$ false positive rate which should be required for treatment studies.

\section{REFERENCES}

1. Krachmer JH, Feder RS, Belin MW. Keratoconus and related noninflammatory corneal thinning disorders. Surv Ophthalmol. 1984;28(4):293-322.
2. Rabinowitz YS. Keratoconus. Surv Ophthalmol 1998;42(4): 297-319.

3. Dawson DG, Randleman JB, Grossniklaus HE, et al. Corneal ectasia after excimer laser keratorefractive surgery: histopathology, ultrastructure and pathophysiology. Ophthalmology 2008; 115(12):2181-2191.

4. Binder PS, Lindstrom RL, Stulting RD, et al. Keratoconus and corneal ectasia after LASIK. J Refract Surg 2005;21(6): 749-752.

5. Seiler T, Koufala K, Richter G. Iatrogenic keratectasia after laser in situ keratomileusis. J Refract Surg 1998;14(3):312-317.

6. Randleman JB, Woodward M, Lynn MJ, Stulting RD. Risk assessment for ectasia after corneal refractive surgery. Ophthalmology 2008;115(1):37-50.

7. Belin MW, Khachikian SS. New devices and clinical implications for measuring corneal thickness [editorial]. Clin Exp Ophthalmol 2006;34(8):729-731.

8. Raiskup-Wolf F, Hoyer A, Spoerl E. Collagen cross-linking with riboflavin and ultraviolet: a light in keratoconus: long-term results. J Cataract Refract Surg 2008;34(5):796-801.

9. Henriquez MA, Izquierdo L Jr, Bernilla C, Zakrzewski PA, Mannis M. 'Riboflavin/Ultraviolet a corneal collagen crosslinking for the treatment of keratoconus: visual outcomes and Scheimpflug analysis.' Cornea 2011;30(3):281-286.

10. Ambrosio R Jr, Luz A, Lopes B, Ramos I, Belin MW. 'Enhanced ectasia screening: the need for advanced and objective data.' $J$ Refract Surg 2014;30(3):151-152.

11. Ramos IC, Correa R, Guerra FP, et al. Variability of subjective classifications of corneal topography maps from LASIK candidates. J Refract Surg 2013;29(11):770-775.

12. Amsler M. 'Le keratocone fruste au javal' Ophthalmologica 1938;96(2):77-83.

13. Amsler M. 'Keratocone classique et keratocone fruste, arguments unitaires. Ophthalmologica 1946;111(2-3):96-101.

14. Krumeich JH, Daniel J, Knülle A. Live-epikeratophakia for keratoconus. J Cataract Refract Surg 1998 April;24(4): 456-463.

15. Rabinowitz Y, Rasheed K. 'KISA\% index: a quantitative videokeratography algorithm embodying minimal topographic criteria for diagnosing keratoconus'. J Cataract Refract Surg 1999;25(10):1327-1335.

16. Maeda N, Klyce S, Smolek M. Comparison of methods for detecting keratoconus using videokeratography. Arch Ophthalmol 1995;113(7):870-874.

17. Maeda N, Klyce S, Smolek M, Thompson H. 'Automated keratoconus screening with corneal topography analysis'. Invest Ophthalmol Vis Sci 1994;35(6):2749-2757.

18. Rabinowitz YS, Garbus J, McDonnell PJ. 'Computer-assisted corneal topography in family members of patients with keratoconus'. Arch Ophthalmol 1990;108(3):365-371.

19. Pinero D, Nieto J, Lopez-Miguel A. 'Characterization of corneal structure in keratoconus'. J Cataract Refract Surg 2012;38(12):2167-2183.

20. Ambrosio R Jr, Belin MW. 'Imaging of the Cornea: topography vs tomography.' J Refract Surg 2010:26(11):847-849.

21. Gilani F, Cortese M, Ambrosio RR Jr, et al. Comprehensive anterior segment normal values generated by rotating Scheimpflug tomography. J cataract Refract Surg 2013; 39(11):1707-1712.

22. Henriquez MA, Izquierdo L Jr, Mannis MJ. Inter eye asymmetry detected by Scheimpflug imaging in subjects with normal corneas and keratoconus. Cornea 2013;32(6):779-782. 
23. Correia FF, Ramos I, Lopes B, et al. Topometric and tomographic indices for the diagnosis of keratoconus. Int J Kerato Ect Cor Dis 2012;1(2):92-99.

24. Ambrosio R Jr. Simplifying ectasia screening with Pentacam corneal tomography. Highlights of Oph Journal 2010;38(3):12-20.

25. Hashemi H1, Beiranvand A, Khabazkhoob M, et al. 'Prevalence of keratoconus in a population-based study in Shahroud.' Cornea. 2013 Nov;32(11):1441-1445.
26. Koller T, Mrochen M, Seiler T. 'Complication and failure rates after corneal cross-linking.' J Cataract Refract Surg 2009 Aug;35(8):1358-1362.

27. Krumeich JH, Daniel J, Knulle A. 'Live-epikeratophakia for keratoconous. J Cataract Refract Surg.’ 1998;24(4):456-463.

28. Salomao MQ, Guerra FP, Ramos IC, et al. 'Accuracy of topometric indices for distinguishing between keratoconic and normal corneas.' Int J Kerat Ect Cor Dis 2013;2(3):108-112. 